\title{
ALMACENAMIENTO DE CARBONO COMO SERVICIO AMBIENTAL EN TRES RESERVAS NATURALES DEL ECUADOR
}

\author{
CARBON STORAGE AS ENVIRONMENTAL SERVICE IN THREE NATURAL \\ RESERVES OF ECUADOR
}
IVÁN FERNANDO PALACIOS OREJUELA ${ }^{1}$, BETTY SELENA CASTRO BENAVIDES ${ }^{2}$, FABIÁN RODRÍGUEZ ESPINOSA ${ }^{3,4}$

1,2 CARRERA DE INGENIERÍA GEOGRÁFICA Y DEL MEDIO AMBIENTE, DEPARTAMENTO DE CIENCIAS DE LA TIERRA Y LA CONSTRUCCIÓN, UNIVERSIDAD DE LAS FUERZAS ARMADAS “ESPE”. Av. General Rumiñahui s/n. Sangolquí, Ecuador. Email: ifpalacios@espe.edu.ec; bscastro1@espe.edu.ec.

${ }^{3}$ GRUPO DE INVESTIGACION GEOESPACIAL, DEPARTAMENTO DE CIENCIAS DE LA TIERRA Y LA CONSTRUCCIÓN, UNIVERSIDAD DE LAS FUERZAS ARMADAS "ESPE". Av. General Rumiñahui s/n. Sangolquí, Ecuador. Email: ffrodriguez3@espe.edu.ec.

${ }^{4}$ FACULTAD DE ECONOMÍA, PONTIFICIA UNIVERSIDAD CATÓLICA DEL ECUADOR. Av. 12 de Octubre 1076 y Roca, Quito, Ecuador.

Recibido: 22 de enero de 2019 / Aceptado: 21 de mayo de 2019

\begin{abstract}
RESUMEN
Las reservas naturales son importantes para la conservación y provisión de servicios ambientales. En Ecuador, estos servicios han sido poco estudiados, especialmente en lo referido al almacenamiento de carbono, significativo por su rol en la mitigación del cambio climático. En 2012 el Ministerio del Ambiente (MAE) realizó una aproximación de la zonificación de los ecosistemas y biomasa (Ton C/ha) que estos almacenan; sin embargo, no se cuenta con información actualizada de la variación que sufre el área ocupada por los ecosistemas, lo que es necesario para la planificación de políticas ambientales. El objetivo del trabajo fue determinar el cambio del valor económico del servicio ambiental por almacenamiento de carbono en tres reservas naturales del Ecuador entre 2012 y 2018, mediante imágenes satelitales. Se consideró al bosque protector Kutukú - Shaimi, y las reservas ecológicas Tapichalaca y Buenaventura como casos de estudio. Primeramente, se calculó la biomasa de cada reserva natural según los datos del MAE. La cobertura vegetal actual se obtuvo con imágenes satelitales Sentinel 2A, a las que se realizó una clasificación supervisada que determinó la dinámica espacial de las nuevas áreas ocupadas por los ecosistemas y posteriormente se calculó la biomasa al año 2018. Se asignó el valor económico al servicio ambiental con el precio promedio de los CER's correspondiente al periodo de tiempo del estudio. Los resultados mostraron un aumento del valor económico por almacenamiento de carbono en las tres reservas naturales, con una diferencia de USD \$2045905.43, obtenida con los datos actualizados. También se evidenció una disminución del área de bosque intervenido en las reservas de Tapichalaca y Buenaventura, lo que indica un proceso de regeneración del bosque. En el bosque protector Kutukú - Shaimi se identificó una disminución del bosque piemontano, lo que implica en la necesidad de evaluar otros servicios ambientales que podrían ser afectados.
\end{abstract}

Palabras clave: bosque protector, imágenes satelitales, regeneración del bosque, reservas ecológicas, valor económico. 


\begin{abstract}
Natural reserves are important for the conservation and provision of environmental services. In Ecuador, these services have been poorly studied, especially the carbon storage, which is relevant for its role in the climate change mitigation. In 2012, the Ministry of the Environment (MAE) made an approximation of the ecosystems zoning and the biomass that they storage (Ton C/ha); However, there is no updated information related to the variation of the occupied area by the ecosystems, which is necessary for planning environmental policies. The aim of the study was to determine the change of the economic value of the carbon storage as environmental service in three natural reserves of Ecuador between 2012 and 2018, through satellite images. The protected forest Kutukú - Shaimi, and the ecological reserves Tapichalaca and Buenaventura were considered as cases of study. Firstly, the biomass of each natural reserve was calculated according to the MAE data. The current vegetation cover was obtained with Sentinel 2A satellite images, a supervised classification was preformed to determine the spatial dynamics of the new areas occupied by the ecosystems and subsequently the biomass was calculated for 2018 . The economic value was assigned to the environmental service with the average price of CER's corresponding to the period of time of the study. The results showed an increase in the economic value of carbon storage in all the reserves, with a difference of USD \$2045905.43, obtained with the updated data. There was also a decrease in the area of the intervened forest in the reserves of Tapichalaca and Buenaventura, which indicates a process of forest regeneration. In the protected forest Kutukú - Shaimi a decrease of the piedmont forest was identified, which indicates the need of determinate what other environmental services could be affected.
\end{abstract}

Keywords: protected forest, satellite images, forest regeneration, ecological reserves, economic value.

\title{
INTRODUCCIÓN
}

Las reservas naturales son actualmente una de las formas más importantes para la conservación de la biodiversidad, ya que, además de ser el hábitat de especies vegetales, animales, y comunidades humanas, éstas reservas brindan servicios ambientales, como por ejemplo la provisión y cuidado de fuentes hídricas, purificación del aire, conservación de la biodiversidad, almacenamiento de carbono, ecoturismo, entre otros (Badola \& Hussain, 2005; Ninan \& Inoue, 2013).

En Ecuador, las reservas naturales gubernamentales forman parte del Sistema Nacional de Áreas Protegidas (SNAP), que en total cubren el 33.26\% del territorio nacional, con lo cual, es el segundo país en América Latina con mayor porcentaje de territorio bajo protección (MAE, 2014). Además, Ecuador es uno de los 17 países mega diversos del planeta (Morillo, 2016); posee la mayor concentración de biodiversidad por kilómetro cuadrado gracias a su ubicación privilegiada sobre la línea ecuatorial y a la presencia de la cordillera de Los Andes, facilitan la existencia diferentes pisos climáticos que albergan distintos ecosistemas, por lo que varias organizaciones no gubernamentales fueron creadas con el objetivo de proteger, conservar y estudiar los servicios y recursos naturales de áreas con importancia crítica que no estén bajo el régimen de protección del SNAP (Fundación Jocotoco, 2018). 
El estudio de los servicios ambientales en el país se ha enfocado principalmente al uso recreativo o ecosistémico (Espinosa, Rodríguez \& Cartuche, 2016; Zafrir \& Rodríguez, 2014), beneficios estéticos (Vallejo \& Rodríguez, 2015), costos de oportunidad en cuanto a casería por grupos indígenas (Cueva, Ortíz \& Jorgenson, 2004; De la Montaña, 2013; Espinosa, Branch \& Cueva, 2014; Zapata, 2001; Zapata, Urgilés \& Suárez, 2009), y pocas investigaciones relacionadas con el almacenamiento de carbono (Ayala, Villa, Aguirre \& Aguirre, 2014; Cadena, Tapia \& Rodríguez, 2017; Jadán, Torres \& Günter, 2012). A pesar de los trabajos realizados, aún persiste una falta de información que permita conocer el papel que juegan las reservas naturales del país en la prestación de servicios ambientales, especialmente al almacenamiento de carbono, debido al reconocimiento que se le ha dado a este servicio en la mitigación del cambio climático (Magnago et al., 2015; Gibbs, Brown, Niles \& Foley, 2007). A nivel mundial, se han realizado muchos estudios que demuestran la importancia social, ambiental y económica que cumplen los bosques en el almacenamiento de carbono (Bechara et al., 2016; Birch et al., 2010; Chazdon et al., 2016; Harmon, Ferrell \& Franklin, 1990; Lewis, Lopez \& Wöll, 2009; Mukul, Herbohn \& Firn, 2016; Ordóñez, de Jong \& Masera, 2001; Yepes et al., 2015), por mencionar algunos.

Basados en las metas y objetivos del protocolo de Kioto, los países desarrollados implementaron proyectos y programas de reducción de emisiones (Ramírez, Rodríguez, Finegan, \& Gómez, 1999). Además, las Naciones Unidas lanzó la iniciativa REED+, con el fin de mantener las reservas de carbono y generar beneficio económico a los países en vías de desarrollo. En el caso de Ecuador, existe un gran potencial para el almacenamiento de carbono, ya que si únicamente se consideran las áreas protegidas amazónicas, se almacenan 708 millones toneladas de carbono (MAE, 2014). Para determinar estas reservas de carbono y su cambio a través del tiempo, una variable importante a considerar es la cubierta vegetal, y una solución práctica para establecerla es mediante el uso de teledetección (Aguas, 2016; Anaya, Chuvieco \& Palacios, 2009; Brown, 2002; Castro, 2013; Dong et al., 2003; Goetz \& Dubayah, 2014; Hansen et al., 2008; Honorio \& Baker, 2010; Patenaude, Milne \& Dawson, 2005; Zhao, Popescu \& Nelson, 2009).

Por su parte, en Ecuador, el Ministerio de Ambiente (MAE) ha trabajado desde el 2008 en programas como Socio Bosque que continua hasta la presente fecha (MAE, 2018), con el cual busca la conservación y protección de sus bosques nativos, páramos u otra vegetación nativa, mediante incentivos económicos a campesinos y comunidades indígenas que se comprometan voluntariamente al programa. Además, el MAE se ha enfocado en la evaluación del estado de los bosques para definir en primera instancia, los ecosistemas que los forman y estudiar el estado del almacenamiento de carbono en los mismos (MAE, 2014), para lo cual utilizó imágenes satelitales (Landsat 8 en su mayoría, Aster y Rapideye), de los años 2010 - 2012 (MAE, 2013). Sin embargo, la zonificación de los ecosistemas obtenidas por el MAE se mantiene a la fecha actual, por lo que no se tiene información actualizada de las áreas que conforman los ecosistemas y consecuentemente de variación de sus reservas de carbono, esto genera respuestas 
políticas desinformadas en torno a este servicio ambiental cuya importancia social, ambiental y económica ha sido justificada anteriormente.

El objetivo del presente estudio fue determinar la variación del valor económico por almacenamiento de carbono en tres reservas naturales del Ecuador entre los años 2012 2018, caso de estudio: bosque protector Kutukú - Shaimi y las reservas ecológicas Tapichalaca y Buenaventura.

\section{METODOLOGÍA}

\section{ÁREA DE ESTUDIO}

\section{Bosque protector Kutukú - Shaimi (BPKS)}

El BPKS fue creado el 10 de julio de 1990, se encuentra al suroriente del Ecuador, en la provincia de Morona Santiago, al oriente de la Cordillera de los Andes, en lo que se conoce como la Cordillera del Kutukú, cuyo nombre proviene de dicha formación geológica (Brito \& Muñoz, 2011) (ver Figura 1). Posee alturas de hasta $2500 \mathrm{msnm}$, con precipitaciones anuales que varían entre 2000 a $3000 \mathrm{~mm}$ y temperaturas de $17^{\circ} \mathrm{C}$ a $22^{\circ} \mathrm{C}$ (MAE, 2012).

La cordillera del Kutukú forma parte de las 25 "zonas calientes" para la conservación de la biodiversidad mundial (Mittermeier, Myers, \& Mittermeier, 1999), que gracias a sus factores ambientales, geológicos e históricos, presenta altos niveles de endemismo y biodiversidad (Gentry, 1995). En total se estima la presencia documentada de por lo menos 480 especies de aves, 51 especies de mamíferos, 81 especies en anfibios, y 41 especies en reptiles (MAE, 2012), lo que destaca su importancia científica. El BPKS también sobresale por ser el bosque protector más grande del Ecuador, con 342025.48 ha aproximadamente, en cuyos territorios se asientan 117 comunidades, en su mayoría de la nacionalidad shuar y el resto mestizas (MAE, 2012).

\section{Reserva ecológica Buenaventura}

La reserva ecológica Buenaventura está ubicada en la provincia de El Oro y protege áreas de bosque nublado piemontano del chocó ecuatoriano por lo que alberga gran cantidad de especies de flora y fauna. Fue creada en el año de 1999 para la protección del Perico de Orces (ver Figura 1). El cambio de la cobertura vegetal a pastizales redujo esta área significativamente en los años 90 , por lo que se trata de un bosque secundario con $5 \%$ a $10 \%$ de bosque natural (Fundación Jocotoco, 2018). Esta reserva se encuentra entre los $400 \mathrm{msnm}$ a $1200 \mathrm{msnm}$, con una temperatura promedio de $14^{\circ} \mathrm{C}$ a $26{ }^{\circ} \mathrm{C}$ y una precipitación anual de 1100 mm (Granda , 2007).

Esta reserva tiene gran importancia biológica ya que alberga a más de 330 especies de aves, 12 de ellas son globalmente amenazadas, mientras que 34 especies son endémicas regionales. Algunos mamíferos registrados son el perezoso de dos dedos de occidente, el oso hormiguero de occidente, el tigrillo chico manchado y pumas. También están 
registradas 41 especies de reptiles y anfibios, de las que un tercio son endémicas y cinco están amenazadas (Fundación Jocotoco, 2018).

\section{Reserva ecológica Tapichalaca}

La reserva Tapichalaca está ubicada en la provincia de Zamora Chinchipe. Debido a la topografía muy inclinada el bosque es denso, musgoso y es hogar de una gran diversidad de especies de flora y fauna (ver Figura 1). Fue creada para proteger el hábitat de la Galaria Jocotoco, ave que dio nombre a la fundación que maneja las reservas; consta de aproximadamente 3500 ha, que cubren zonas de páramo, bosque subtropical andino y bosque nublado de montaña frío y húmedo, este último tiene un rango altitudinal de 2200 msnm a $3400 \mathrm{msnm}$, con una temperatura entre $10^{\circ} \mathrm{C}$ a $20^{\circ} \mathrm{C}$ y una precipitación anual que puede alcanzar los $5000 \mathrm{~mm}$. La regeneración del bosque es parte de un ciclo de deslizamientos de tierras producto de la geología inestable del lugar, donde la fundación realiza programas de reforestación con especies nativas.

Esta reserva se localiza en una región de gran biodiversidad y endemismo. La reserva protege 130 especies de plantas endémicas del Ecuador, de las cuales 50 son endémicas de la localidad. El bosque es hogar de más de 300 especies de aves y alberga poblaciones de tapir andino y oso de anteojos, además protege 7 especies de mamíferos vulnerables, cuatro casi amenazados (Fundación Jocotoco, 2018).

\section{ESTIMACIÓN DE LA COBERTURA VEGETAL: AÑO 2012}

Para determinar la cobertura vegetal, se basó en la información oficial del MAE, la cual fue generada a partir de factores diagnósticos, de tipo biótico y abiótico, que incluyen temperatura, precipitación y geoformas, entre otros. Con estos factores se desarrollaron clasificaciones jerárquicas, modelamiento biofísico, interpretación de imágenes satelitales $(2010$ - 2012) y validación en campo que permitieron predecir la distribución de los ecosistemas. Del proceso anterior, el MAE generó el Mapa de Ecosistemas de Ecuador Continental al año 2012 (MAE, 2013). Con esta información base, se procedió a extraer los ecosistemas de las tres áreas protegidas bajo estudio, como se observa en la Figura 1. 


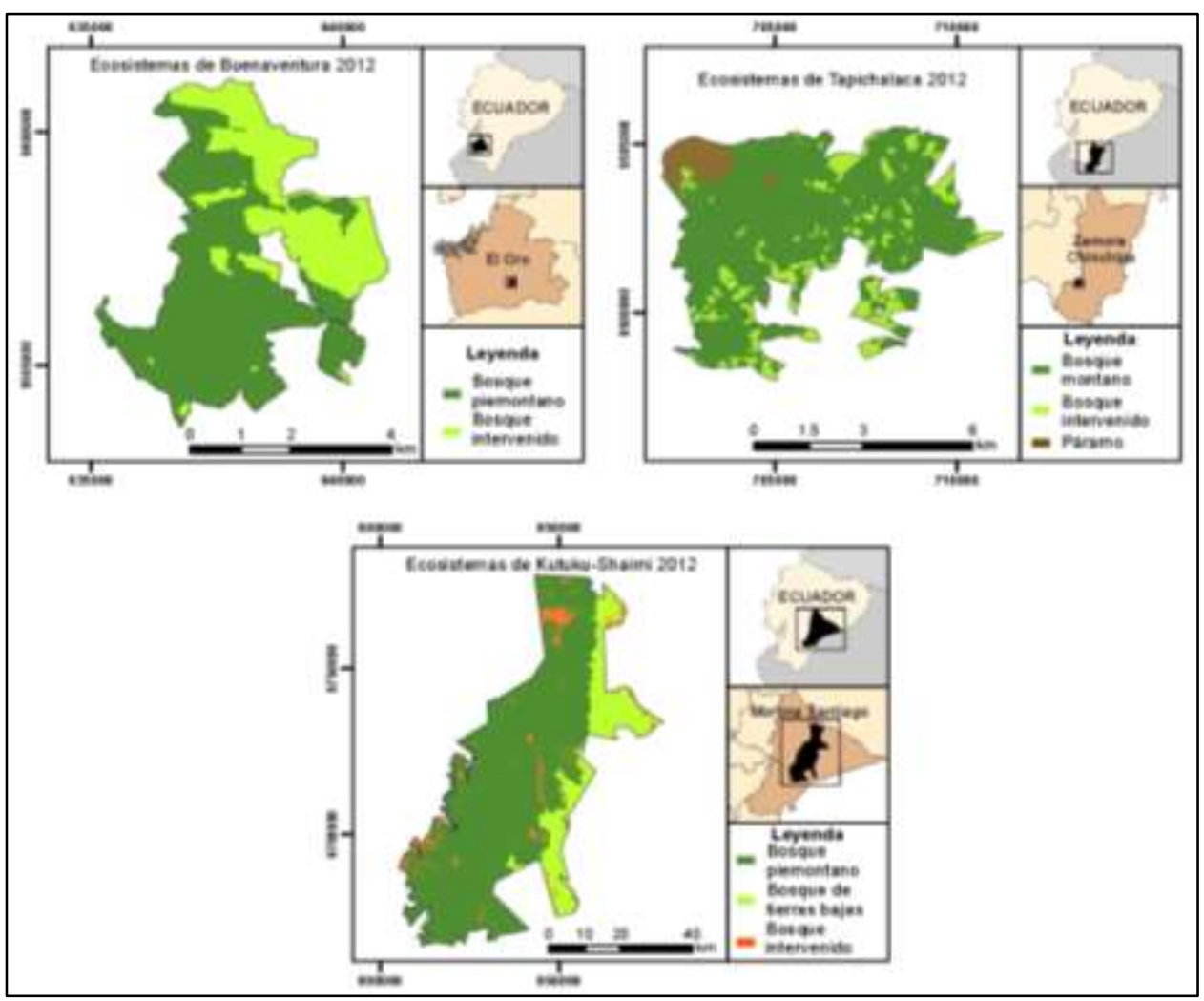

Figura 1. Ubicación y ecosistemas del año 2012 de las reservas naturales bajo estudio (MAE, 2013) .

Una vez identificados los ecosistemas de cada reserva natural, se calculó la superficie ocupada por cada ecosistema mediante una herramienta dentro de un sistema de información geográfica. En las tres áreas protegidas se consideró las coberturas vegetales de mayor superficie, ya que éstas aportarán principalmente al almacenamiento de carbono. La información se resume en la Tabla 1 a continuación:

Tabla 1. Superficie ocupada por los ecosistemas en cada reserva natural.

\begin{tabular}{|c|c|c|c|c|}
\hline Bosque & Ecosistema & $\begin{array}{l}\text { Área (ha) } \\
\text { año } 2012\end{array}$ & $\begin{array}{l}\text { Área (ha) } \\
\text { año } 2018\end{array}$ & $\begin{array}{c}\text { Área total } \\
\text { (ha) } \\
\text { Año } 2018\end{array}$ \\
\hline \multirow{3}{*}{$\begin{array}{l}\text { Kutukú - } \\
\text { Shaimi }\end{array}$} & $\begin{array}{l}\text { Bosque siempreverde piemontano de las } \\
\text { cordilleras del Cóndor - Kutukú }\end{array}$ & 267425.39 & 232957.62 & \multirow{3}{*}{342025.48} \\
\hline & $\begin{array}{l}\text { Bosque siempreverde de tierras bajas del } \\
\text { Abanico del Pastaza }\end{array}$ & 59423.15 & 104039.49 & \\
\hline & Intervenido & 15176.94 & 5028.37 & \\
\hline \multirow{3}{*}{ Tapichalaca } & $\begin{array}{l}\text { Bosque siempreverde montano del Sur de la } \\
\text { Cordillera Oriental de los Andes }\end{array}$ & 3015.95 & 3422.82 & \multirow{3}{*}{3826.96} \\
\hline & intervenido & 606.96 & 166.57 & \\
\hline & $\begin{array}{l}\text { Arbustal siempreverde y Herbazal del } \\
\text { Páramo }\end{array}$ & 204.06 & 237.57 & \\
\hline \multirow{2}{*}{ Buenaventura } & Bosque siempreverde piemontano del & 1409.73 & 1520.97 & \multirow{2}{*}{2100.94} \\
\hline & Intervenido & 691.21 & 579.97 & \\
\hline
\end{tabular}


$\mathrm{Al}$ interpretar los resultados de las coberturas vegetales predominantes, se identificaron áreas intervenidas en todas las zonas de estudio, las cuales corresponden a zonas usadas como pastizales para ganado (bosque Kutukú - Shaimi), y zonas nuevas incorporadas que corresponden a un bosque secundario (reserva Tapichalaca y Buenaventura).

\section{ESTIMACIÓN DE LA COBERTURA VEGETAL: AÑO 2018}

Los insumos para el cálculo de la cobertura vegetal fueron imágenes satelitales Sentinel 2A del año en cuestión, con una resolución espacial de $10 \mathrm{~m}$, suficiente al considerar la extensión de las áreas de estudio.

Se tomó como base la delimitación de propuesta por MAE (2013), para determinar de manera inicial los ecosistemas. Sobre las imágenes satelitales, se realizó un muestreo de las diferentes coberturas vegetales que contienen información de la respuesta espectral de la cubierta vegetal en las diferentes bandas utilizadas, con las cuales, se evaluó la separabilidad de las muestras antes de realizar la clasificación. Por último, se realizó una clasificación supervisada en cada reserva natural, cuyo resultado se observa en la Figura 2 .

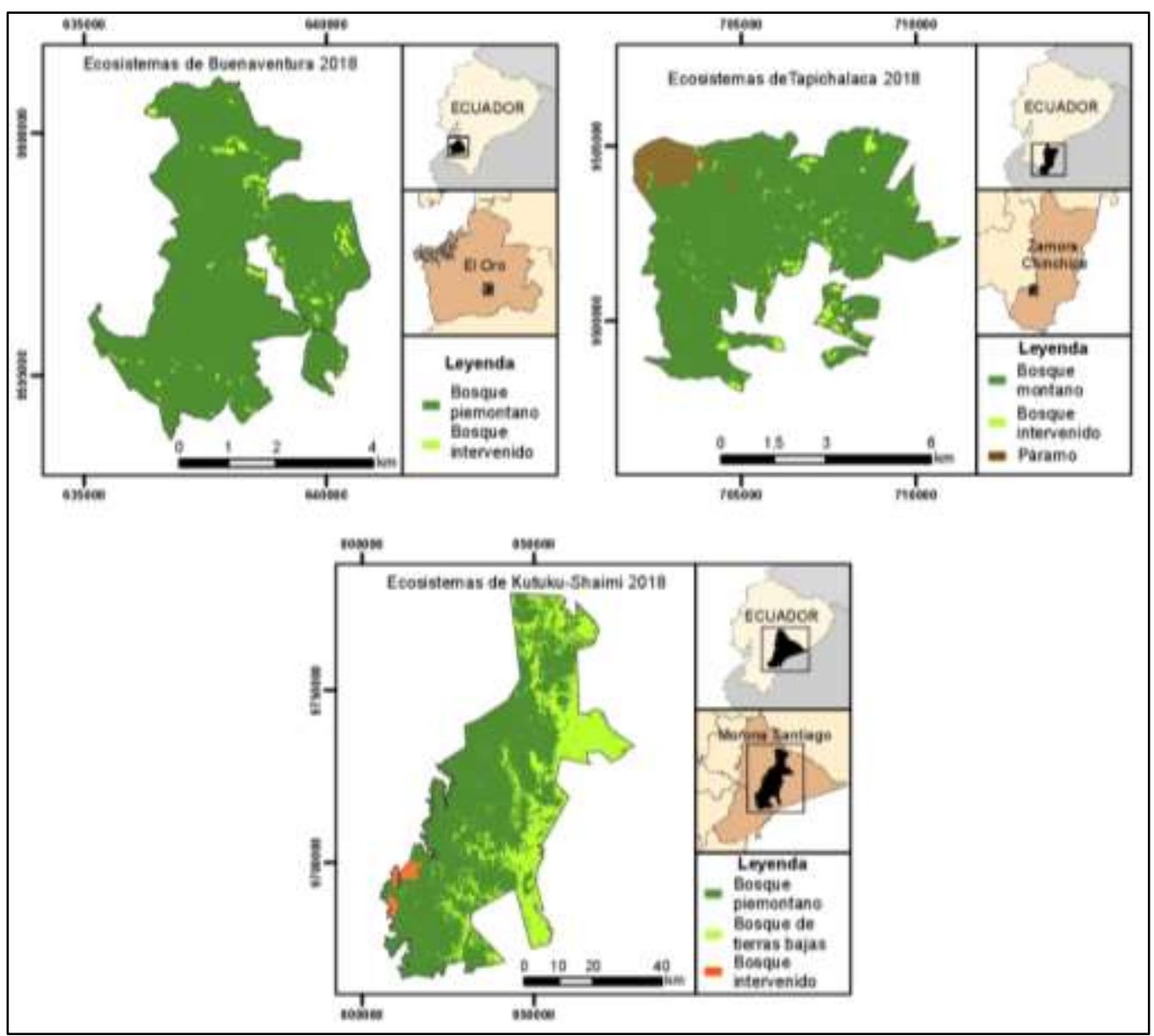

Figura 2. Ubicación y ecosistemas del año 2018 de las reservas naturales bajo estudio. 


\section{VALORACIÓN ECONÓMICA}

El valor de almacenamiento de carbono se obtuvo del Paquete de Informe sobre los Bosques 2015 del Ecuador, realizado por la FAO (2015) (Food and Agriculture Organization) y otras organizaciones internacionales relacionadas al tema en cuestión, del cual se tomó el valor únicamente de los bosques existentes en cada reserva natural, debido a que estas coberturas vegetales constan en dicho documento. Estos valores de almacenamiento de carbono fueron calculados en Ton $\mathrm{C} / \mathrm{ha}$.

Adicionalmente, se utilizó el método de trasferencia de conocimiento en cada reserva natural para completar los valores de las coberturas vegetales faltantes. El estudio de Jadán, Torres \& Günter (2012), propone un valor de 33.20 Ton C/ha, para una cobertura vegetal de pastizales en el Parque Nacional Sumaco, cuyas condiciones se asemeja al BPKS; Ayala, Villa, Aguirre \& Aguirre (2014), indican un almacenamiento de 116.18 Ton C/ha correspondiente a los páramos del Parque Nacional Yacurí, el mismo que fue utilizado como valor de referencia en la reserva Tapichalaca. Por último, Spracklen \& Righelato (2016), realizaron estudios en los bosques secundarios (área intervenida) de Tapichalaca y Buenaventura, donde determinaron valores de 18.30 y 2.70 Ton C/ha respectivamente.

El almacenamiento de carbono se puede trasformar en valores monetarios, gracias a que existen mercados internacionales que apoyan este tipo de servicio ambiental en pro de la conservación de áreas naturales y lucha contra el cambio climático, como el CER (Certified Emission Reductions) y EUA (European Union Allowances), entre los más importantes (SENDECO2, 2018). Según el Banco Mundial, los precios en las iniciativas implementadas se encuentran entre $1-135$ USD/Ton $\mathrm{CO}_{2}$, y de estos, el $46 \%$ de las emisiones son cubiertas con precios menores a $10 \mathrm{USD} / \mathrm{Ton} \mathrm{CO}_{2}$ (Word Bank Group, 2018). También, SENDECO2 (2018), en su página oficial, establece el precio anual de carbono dado por la EUA de USD \$ 16.96 y en el CER de USD \$ 0.26 por tonelada de $\mathrm{CO}_{2}$ emitido. En el país, según la Evaluación Nacional Forestal se presentó un precio de 7.3 USD/Ton $\mathrm{CO}_{2}$ (MAE, 2014), sin embargo, no se puede tomar ese valor ya que no es constante en el tiempo debido a la fluctuación del precio en los mercados de carbono. Además, el Ecuador no cumple los requisitos para el mercado de la EUA, pero sí en el CER, por lo que, se consideró un precio promedio desde el año 2012 hasta el 2018 en base al CER para el cálculo de este servicio ambiental, cuyo valor medio fue de 0.77 USD/Ton $\mathrm{CO}_{2}$. Mediante la Ecuación 1 se obtuvo el valor económico del servicio ambiental estudiado, como sigue:

$$
B_{c}=Q * P * a
$$

Donde:

$B_{c}$ : valor económico de los beneficios por almacenamiento de carbono;

$Q$ : cantidad de toneladas de carbono almacenado por cada hectárea de cobertura vegetal;

$P$ : precio de tonelada de carbono almacenado en USD por tonelada de carbono;

$a$ : hectáreas de cobertura vegetal calculadas. 


\section{RESULTADOS Y DISCUSIÓN}

Los precios calculados por almacenamiento de carbono en las tres reservas de estudio se resumen a continuación en la Tabla 2 .

Tabla 2. Valor monetario obtenido entre los años 2012 - 2018 en las reservas naturales.

\begin{tabular}{|c|c|c|c|c|c|c|}
\hline \multirow[b]{2}{*}{ Bosque } & \multirow[b]{2}{*}{ Ecosistema } & \multicolumn{2}{|c|}{ Área (ha) } & \multirow{2}{*}{$\begin{array}{c}\text { Carbono } \\
\text { (Ton } \\
\text { C/ha) } \\
\end{array}$} & \multicolumn{2}{|c|}{ Valor monetario (USD) } \\
\hline & & 2012 & 2018 & & $2012 *$ & 2018* \\
\hline \multirow{3}{*}{ 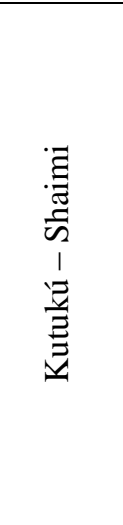 } & $\begin{array}{l}\text { Bosque } \\
\text { siempreverde } \\
\text { piemontano de } \\
\text { las cordilleras } \\
\text { del Cóndor - } \\
\text { Kutukú } \\
\text { Bosque }\end{array}$ & 267425.39 & 232957.62 & $\begin{array}{c}\text { (a) } \\
122.77\end{array}$ & 25374302.84 & 22103874.27 \\
\hline & $\begin{array}{ll} & \\
\text { siempreverde de } \\
\text { tierras bajas del } \\
\text { Abanico del } \\
\text { Pastaza }\end{array}$ & 59423.15 & 104039.49 & $\begin{array}{c}\text { (a) } \\
160.40\end{array}$ & 7366467.19 & 12897389.14 \\
\hline & Intervenido & 15176.94 & 5028.37 & $\begin{array}{c}\text { (b) } \\
33.20\end{array}$ & 389422.94 & 129022.23 \\
\hline \multirow{3}{*}{ 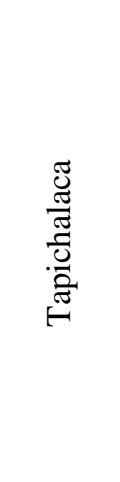 } & $\begin{array}{l}\text { Bosque } \\
\text { siempreverde } \\
\text { montano del Sur } \\
\text { de la Cordillera } \\
\text { Oriental de los } \\
\text { Andes }\end{array}$ & 3015.95 & 3422.82 & $\begin{array}{c}\text { (a) } \\
123.10\end{array}$ & 286933.13 & 325642.22 \\
\hline & Intervenido & 606.96 & 166.57 & $\begin{array}{c}(\mathrm{d}) \\
18.30\end{array}$ & 8584.41 & 2355.90 \\
\hline & $\begin{array}{l}\text { Arbustal } \\
\text { siempreverde y } \\
\text { Herbazal del } \\
\text { Páramo }\end{array}$ & 204.06 & 237.57 & $\begin{array}{c}\text { (c) } \\
116.18\end{array}$ & 18322.39 & 21331.54 \\
\hline \multirow{2}{*}{ 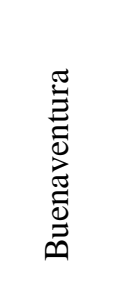 } & $\begin{array}{l}\text { Bosque } \\
\text { siempreverde } \\
\text { piemontano del } \\
\text { Catamayo - } \\
\text { Alamor }\end{array}$ & 1409.73 & 1520.97 & $\begin{array}{c}\text { (a) } \\
122.77\end{array}$ & 133760.07 & 144315.22 \\
\hline & Intervenido & 691.21 & 579.97 & $\begin{array}{c}\text { (d) } \\
2.70\end{array}$ & 1442.36 & 1210.24 \\
\hline
\end{tabular}

* $\overline{\text { El valor promedio usado de almacenamiento de carbono según el Banco mundial, se puede consultar en }}$ https://www.sendeco2.com/es/precios-co2. El valor promedio del carbono almacenado (Ton) por hectárea de ecosistema se obtuvo de las siguientes fuentes: (a) FAO (2015), (b) Jadán, Torres \& Günter (2012), (c) Ayala, Villa, Aguirre \& Aguirre (2014) y (d) Spracklen \& Righelato (2016).

Al analizar los resultados obtenidos entre los años 2012 - 2018, se identificó que en todas las reservas naturales ha existido un cambio evidente en la extensión de las coberturas vegetales. En el caso del BPKS, el Bosque siempreverde piemontano de las cordilleras del Cóndor - Kutukú se redujo un 12.89\%, lo que indica un retroceso o cambio en la cobertura vegetal; el área intervenida se redujo $17.08 \%$, que señala una recuperación de 
áreas que antes eran usadas para la ganadería, mientras que Bosque siempreverde de tierras bajas del Abanico del Pastaza se incrementó $42.88 \%$, por lo que se infiere que este tipo de bosque ahora ocupa otras áreas que fueron intervenidas o de tipo piemontano.

En cuanto a la reserva ecológica Tapichalaca, el Bosque siempreverde montano del Sur de la Cordillera Oriental de Los Andes, incrementó su superficie en 11.89\%; el área intervenida se redujo $72.56 \%$, y el Arbustal siempreverde y Herbazal del Páramo incrementó su área un $14.11 \%$, lo que señala un resultado positivo gracias a la incorporación y cuidado de la nueva área intervenida a la reserva ecológica.

Por su parte, la reserva ecológica Buenaventura registró en el Bosque siempreverde piemontano del Catamayo - Alamor un incremento de $7.31 \%$ en su superficie, y una reducción del área intervenida de $16.09 \%$, porcentajes que revelan una mejora considerable en la cobertura vegetal de la reserva en mención.

El valor económico calculado para las tres reservas naturales también presentó diferencias significativas, ya que el precio obtenido con la información utilizada hasta la fecha por el MAE (imágenes del 2010 - 2012), es menor al valor obtenido con la metodología presentada en este estudio (imágenes del 2018). En el BPKS, se incrementó USD \$ 2000092.68 (5.69\%) entre los años comparados. Sin embargo, es importante resaltar la reducción de la cobertura del bosque piemontano, ya que este ecosistema alberga una gran diversidad biológica, y a pesar de existir un aumento del almacenamiento de carbono producto del incremento del bosque siempre verde de tierras bajas, se deberían analizar otros servicios ambientales que podrían estar siendo afectados. Para esto se sugieren medidas de regeneración a escala local que permitan el desarrollo de la cobertura vegetal, como las recomendadas por Chazdon \& Uriarte (2016).

Las reservas de Tapichalaca y Buenaventura presentaron un aumento de USD \$ 35489.73 (10.16\%) y USD \$ 10323.02 (7.09\%) al año 2018 respectivamente. La variación del valor monetario en la reserva Buenaventura puede explicarse, ya que, en el año 2011 se realizó una compra importante de pastizales a incluir en el área de la reserva, por lo que la regeneración del bosque ayudó al aumento de la cobertura vegetal y con esto, al servicio evaluado, a pesar de no ser un bosque muy antiguo. Con estos resultados se demostró que el aumento de la biomasa y consecuentemente del valor económico por almacenamiento de carbono en las reservas bajo estudio, destaca el papel que juegan los bosques restaurados en la mitigación de las emisiones de carbono.

El valor económico final, sugiere que Ecuador, para el año 2018 posee un precio por almacenamiento de carbono de USD \$2045905.43 más que lo obtenido con la información que maneja actualmente el MAE. Además, cabe señalar que el presente estudio fue realizado en 3 reservas naturales, con las que se obtuvo resultados muy prometedores, por lo que sería necesario efectuar un estudio de todo el SNAP, para conocer el estado de conservación actual y determinar si es efectiva la ampliación y mantenimiento de las áreas naturales con sus distintos servicios ambientales que cada una ofrece. 


\section{CONCLUSIONES}

Mediante el uso de imágenes satelitales y la aplicación de clasificación supervisada fue factible comparar y calcular el área actual correspondiente a cada cobertura vegetal y determinar los cambios que han existido entre los años 2012 y 2018, con lo que se demostró la existencia de una variación en la superficie de los ecosistemas en las áreas de estudio, entre la base de datos oficial del país (MAE) y la realidad al presente año.

La diferencia del valor económico por almacenamiento de carbono calculado con la información oficial del MAE y la metodología aplicada en este estudio, resultó en un aumento de USD \$2045905.43, en un periodo de 6 años, ese valor demuestra los potenciales beneficios económicos que pueden ser obtenidos de la conservación de los bosques. Sin embargo, en el BPKS donde el área de bosque piemontano disminuyó es necesario analizar otros servicios ecosistémicos que podrían estar siendo afectados e implementar soluciones que permitan el desarrollo de la cobertura vegetal con una correcta delimitación de los ecosistemas.

\section{REFERENCIAS}

Aguas, C. (2016). Estimación de biomasa aérea forestal en bosque de robedales del antiplano norte de Antioquia, utilizando teledetección. Medellín, Colombia: Tesis de maestría.

Anaya, J., Chuvieco, E., \& Palacios, A. (2009). Aboveground biomass assessment in Colombia: A remote sensing approach. Forest Ecology and Management, 2574, 1237-1246. doi:https://doi.org/10.1016/j.foreco.2008.11.016

Ayala, L., Villa, M., Aguirre, Z., \& Aguirre, N. (2014). Cuantificación del carbono en los páramos del parque nacional Yacurí, provincias de Loja y Zamora Chinchipe, Ecuador. Revista del Centro de Estudios y Desarrollo de la Amazonía, 4(1), 45-52.

Badola, R., \& Hussain, S. (2005). Valuing ecosystem functions: an empirical study on the storm protection function of Bhitarkanika mangrove ecosystem, India. Environmental Conservation, 32(1), 85-92. doi:10.1017/S0376892905001967

Bechara, F., Dickens, S., Farrer, E., Larios, L., Spotswood, E., Mariotte, P., \& Sudind, K. (2016). Neotropical rainforest restoration: Comparing passive, plantation and nucleation approaches. Biodvers. Conserv., 25(11), 2021-2034. doi:10.1007/s10531-016-1186-7

Birch, J., Newton, A., Aquino, C., Cantarello, E., Echeverría, C., Kitzberger, T., \& Garavito, N. (2010). Cost-effectiveness of dryland forest restoration evaluated by spatial analysis of ecosystem services. Proceedings of the National Academy of Sciences of the United States of America, 107, 21925-21930.

Brito, J., \& Muñoz, J. (2011). Línea base del Bosque Protector Cutucú-Shaimi componente fauna. ResearchGate. doi:10.13140/RG.2.2.29204.19847

Brown, S. (2002). Measuring carbon in forests: current status and future challenges. Environmental Pollution, 116(3), 363-372. doi:https://doi.org/10.1016/S0269-7491(01)00212-3

Cadena, P., Tapia, E., \& Rodríguez, F. (2017). Valor económico del almacenamiento de carbono en los páramos de la Reserva Ecológica El Ángel. Revista Geoespacial, (14.1), 65-82.

Castro, D. (2013). Monitoreo de bosques utilizando NDVI Rededge de Rapideye. Revista Geoespacial, (10), 58-71. 
Chazdon, R., \& Uriarte, M. (2016). Natural regeneration in the context of large-scale forest and landscape restoration in the tropics. BIOTROPICA, 48(6), 709-715.

Chazdon, R., Broadbent, E., Rozendaal, D., Bongers, F., Almeyda, A., Aide, M., . . Poorter, L. (2016). Carbon sequestration potential of second-growth forest regeneration in the Latin American tropics. Science Advances, 2(5), 1-10.

Cueva, R., Ortíz, A., \& Jorgenson, J. (2004). Cacería de fauna silvestre en el área de amortiguamiento del Parque Nacional Yasuní, Amazonía Ecuatoriana. Memorias del VI Congreso Internacional sobre Manejo de Fauna Silvestre en la Amazonía y Latinoamérica, 524-539.

De la Montaña, E. (2013). Cacería de subsistencia de distintos grupos indígenas de la Amazonía ecuatoriana. Ecosistemas, 22(2), 84 - 96. doi:10.7818/ECOS.2013.22-2.13

Dong, J., Kaufmann, R., Myneni, R., Tucker, C., Kauppi, P., Liski, J., . . Hughes, M. (2003). Remote sensing estimates of boreal and temperate forest woody biomass: carbon pools, sources, and sinks. Remote Sensing of Environment, 84(3), 393-410. doi:https://doi.org/10.1016/S0034-4257(02)00130-X

Espinosa, J., Rodríguez, F., \& Cartuche, L. (2016). Valoración económica del uso recreativo y ecosistémico del Parque Nacional Podocarpus (PNP), por medio del método de costo de viaje. Revista Geoespacial, (13.2), 1-13.

Espinosa, S., Branch, L., \& Cueva, R. (2014). Road Development and the Geography of Hunting by an Amazonian Indigenous Group: Consequences for Wildlife Conservation. PLoS ONE, 9(12), 1-21. doi:DOI:10.1371/journal.pone.0114916

FAO. (2015). Paquete de Informe sobre los Bosques 2015 del Ecuador. Obtenido de http://suia.ambiente.gob.ec/documents/10179/185860/Reporte_FRA_Ecuador_ago2014. pdf/ac6e9aae-b327-46d4-8640-a7447eaa4153

$\begin{array}{lllll}\text { Fundación } & \text { Jocotoco. } & \text { (2018). Buenaventura. }\end{array}$ http://www.fjocotoco.org/buenaventura2.html

Fundación Jocotoco. (2018). Programa de voluntariado. Obtenido de http://www.fjocotoco.org/voluntarios.html

Fundación Jocotoco. (2018). Tapichalaca. Obtenido de http://www.fjocotoco.org/tapichalaca.html

Gentry, A. (1995). Patterns of diversity and floristic composition in neotropical montane forest. Biodiversity and conservation of neotropical, 103-126.

Gibbs, H., Brown, S., Niles, J., \& Foley, J. (2007). Monitoring and estimating tropical forest carbon stocks: making REDD a reality. Environmental Research Letters, 2(4), 1-13.

Goetz, S., \& Dubayah, R. (2014). Advances in remote sensing technology and implications for measuring and monitoring forest carbon stocks and change. Carbon Management, 2(3), 231-244. doi:https://doi.org/10.4155/cmt.11.18

Granda , T. (2007). Diversidad Orquideológica de la Reserva Ecológica Buenaventura de la parroquia Moromoro, cantón Piñas, provincia de El Oro. Universidad Nacional de Loja. Loja, Ecuador.

Hansen, M., Stehman, S., Potapov, P., Loveland, T., Townshend, J., DeFries, R., . . DiMiceli, C. (2008). Humid tropical forest clearing from 2000 to 2005 quantified by using multitemporal and multiresolution remotely sensed data. PNAS, 105(27), 9439-9444.

Hansen, J., Sato, M., Kharecha, P., Beerling, D., Berner, R., Masson-Delmotte, V., . . Royer, D. Z. (2008). Target atmospheric CO2: Where should humanity aim?. Open Atmos. Sci. J., 2, 217-231. doi:10.2174/1874282300802010217 
Harmon, M., Ferrell, W., \& Franklin, J. (1990). Effects on Carbon Storage of Conversion of OldGrowth Forests to Young Forests. Science, 247(4943), 699-702. doi:10.1126/science.247.4943.699

Honorio, E., \& Baker, T. (2010). Manual para el monitoreo del ciclo del carbono en bosques amazónicos (1st ed.). Lima, Perú: ESERGRAF.

Jadán, O., Torres, B., \& Günter, S. (2012). Influencia del uso de la tierra sobre almacenamiento de carbono en sistemas productivos y bosque primario en Napo, Reserva de Biosfera Sumaco, Ecuador. Revista Amazónica: Ciencia y Tecnología, 1(3), 173-186.

Lewis, S., Lopez, G., \& Wöll, H. (2009). Increasing carbon storage in intact African tropical forests. Nature, 457, 1003-1003.

MAE. (2012). Área de Bosque y Vegetación Protectora KUTUKÚ-SHAMI: Plan de Manejo 20122017. CARE - Ecuador.

MAE. (2013). Mapa de ecosistemas de Ecuador continental. Obtenido de http://mapainteractivo.ambiente.gob.ec/portal/documentos/metadatos/metadato_ecosiste mas_actualizado_26012018.pdf

MAE. (2014). Áreas protegidas del Ecuador socio estratégico para el desarrollo. Quito, Ecuador.

MAE. (2014). Evaluación Nacional Forestal: Resultados. Obtenido de http://suia.ambiente.gob.ec/documents/10179/185860/Evaluaci\%C3\%B3n+Nacional+Fo restal_NREFD+1.pdf/955aaa38-34b6-4b4d-9278-8fe915df893f

MAE. (2018). Resultados de Socio Bosque. Obtenido de http://sociobosque.ambiente.gob.ec/node/44

Magnago, L., Magrach, A., Laurance, W., Martins, S., Meira-Neto, J., Simonelli, M., \& Edwards, D. (2015). Would protecting tropical forest fragments provide carbon and biodiversity cobenefits under REDD+?. Global Change Biology, 21, 3455-3468.

Mittermeier, R., Myers, N., \& Mittermeier, C. (1999). Biodiversidad Amenazada, las Ecorregiones Terrestres Prioritarias del Mundo. Conservation International y Agrupación Sierra Madre.

Morillo, I. (2016). Propuesta de una red de áreas naturales protegidas para el Ecuador continental. Madrid, España. Obtenido de http://eprints.ucm.es/36209/1/T36933.pdf

Mukul, S., Herbohn, J., \& Firn, J. (2016). Co-benefits of biodiversity and carbon sequestration from regenerating secondary forests in the Philippines uplands: Implications for forest landscape restoration. Biotropica, 48, 882-889.

Ninan, K., \& Inoue, M. (2013). Valuing forest ecosystem services: Case study of a forest reserve in Japan. Ecosystem Services, 5, 78-87. doi:https://doi.org/10.1016/j.ecoser.2013.02.006

Ordóñez, J., de Jong, B., \& Masera, O. (2001). Almacenamiento de carbono en un bosque de Pinus pseudostrobus en Nuevo San Juan, Michoacán. Madera y Bosques, 7(2), 27-47. doi:https://doi.org/10.21829/myb.2001.721310

Patenaude, G., Milne, R., \& Dawson, T. (2005). Synthesis of remote sensing approaches for forest carbon estimation: reporting to the Kyoto Protocol. Environmental Science \& Policy, 8(2), 161-178. doi:https://doi.org/10.1016/j.envsci.2004.12.010

Ramírez, O., Rodríguez, L., Finegan, B., \& Gómez, M. (1999). Implicaciones económicas del secuestro de $\mathrm{CO} 2$ en los bosques naturales. Revista Forestal Centroamericana, 279, 1016.

SENDECO2. (2018). Precios CO2. Obtenido de https://www.sendeco2.com/es/precios-co2 
Spracklen, D., \& Righelato, R. (2016). Carbon storage and sequestration of re-growing montane forests in southern Ecuador. Forest Ecology and Management, 364, 139-144. doi:https://doi.org/10.1016/j.foreco.2016.01.001

Vallejo, J., \& Rodríguez, F. (2015). Valoración de los beneficios que genera el Parque Metropolitano de Quito. Revista Geoespacial, (12), 1-14.

Word Bank Group. (2018). State and Trends of Carbon Pricing 2018. Washington DC: ECOFYS

Yepes, A., Herrera, J., Phillips, J., Cabrera, E., Galindo, G., Granados, E., . . Cardona, M. (2015). Contribución de los bosques tropicales de montaña en el almacenamiento de carbono en Colombia. Revista de Biología Tropical, 63(1), 69-82.

Zafrir, R., \& Rodríguez, F. (2014). Valoración económica del uso recreativo de la reserva geobotánica Pululahua (RGP) a través del método de costo de viaje. Revista Geoespacial. (11), 16-27.

Zapata, G. (2001). Sustentabilidad de la cacería de subsistencia: el caso de cuatro comunidades Quichuas en la amazonía nororiental ecuatoriana. Revista Mastozoología Neotropical, $8(1), 59$ - 66.

Zapata, G., Urgilés, C., \& Suárez, E. (2009). Mammal hunting by the Shuar of the Ecuadorian Amazon: is it sustainable? Fauna \& Flora Internationa Oryx, 43(3), 375 - 385. doi:10.1017/S0030605309001914

Zhao, K., Popescu, S., \& Nelson, R. (2009). Lidar remote sensing of forest biomass: A scaleinvariant estimation approach using airborne lasers. Remote Sensing of Environment, 113(1), 182-196. doi:https://doi.org/10.1016/j.rse.2008.09.009 